\title{
Protective Effect of Swimming and Genistein on the Expression of microRNA 132, Insulin Growth Factor 1, and Brain-derived Neurotrophic Factor Genes, as well as Spatial Memory, in the Hippocampus of Diabetic Ovariectomized Rats
}

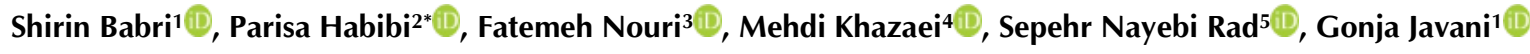 \\ 1 Drug Applied Research Center, Tabriz University of Medical Sciences, Tabriz, Iran \\ 2 Neurophysiology Research Center, Hamadan University of Medical Sciences, Hamadan, Iran \\ ${ }^{3}$ Department of Pharmaceutical Biotechnology, School of Pharmacy, Hamadan University of Medical Sciences, \\ Hamadan, Iran \\ 4 Student Research Committee, Hamadan University of Medical Sciences, Hamadan, Iran \\ ${ }^{5}$ Faculty of Medicine, Tehran University of Medical Sciences, Tehran, Iran
}

*Corresponding author:

Parisa Habibi, Neurophysiology

Research Center, Hamadan University of Medical Sciences, Hamadan, Iran

Tel: +989144194266

Email: pa.habibi@umsha.ac.i

Dr.habibi2007@yahoo.com

Received: 07 Nov. 2020

Accepted: 27 Jan 2021

ePublished: 01 Nov. 2021

\begin{abstract}
Background and Objective: The present study aimed to assess the effects of the combined use of exercise and genistein on the hippocampal expression of microRNA-132, IGF-1, and BDNF in type 2 diabetic ovariectomized rats.

Materials and Methods: Wistar female rats in the weight range of $180-220 \mathrm{gr}(n=10)$ were assigned to six groups: sham, ovariectomy, ovariectomized diabetic, ovariectomized diabetic treated with genistein for eight weeks, diabetic ovariectomized treated with swimming for eight weeks, and a group that was treated with both genistein and swimming for eight weeks. The effect of those treatments was assessed by the determination of microRNA-132, insulin growth factor 1 (IGF-1), and brain-derived neurotrophic factor (BDNF) expression levels within the hippocampus. These genes were evaluated by real-time-polymerase chain reaction (RT-PCR) and spatial memory was assessed by the Morris water maze.

Results: Ovariectomy demonstrated a decrease in the expression of microRNA-132, IGF-1, and BDNF in the hippocampus, as well as spatial memory, in diabetic ovariectomized rats, which showed a greater reduction in the expression of those genes in rats $(P<0.05)$. Nevertheless, genistein administration, swimming training, and a combination of them significantly up-regulated microRNA132, BDNF, and IGF-1 expression, as well as spatial memory $(\mathrm{P}<0.05)$.

Conclusions: As evidenced by the obtained results, the combined use of genistein and swimming could prevent estrogen deficiency effects in the hippocampus of ovariectomized diabetic rats.

Keywords: Diabetes, Genistein, Ovariectomy, Spatial memory, Swimming
\end{abstract}

\section{Background}

The incidence and progression of type 2 diabetes increase the risk of cognitive impairment in postmenopausal women [1]. Some significant features, such as neuronal degeneration, apoptosis, and abnormal neurotransmitter secretion, perform a peculiar role in diabetic neuropathy [2] in menopausal women [3]. It is acknowledged that microRNAs, 18 24 nucleotides in length, play a critical role in the regulation of post-transcriptional gene appearance of target mRNA [4]. MicroRNAs and their target genes contribute greatly to dynamic biological processes, such as neuronal apoptosis and degeneration [5].

The related literature has demonstrated that unbalanced microRNA expression as an unbalanced expression of microRNA-132 is involved in diabetes pathogenesis and menopausal neuropathy [2] [6]. Earlier studies have pointed out that microRNA-132 represses the expression of neurotrophic and growth factors, such as insulinlike growth factor 1 (IGF-1) and Brain-Derived Neurotrophic Factor (BDNF) within the brain [7]. High concentrations of BDNF exist in the cortex and hippocampus, [8] and its altered expression is recognized to play a major role in psychiatric disorders, synaptic plasticity, and the functions correlated with the hippocampus [9]. Furthermore, growth hormone $(\mathrm{GH})$ and IGF-1 have been recently discovered to affect the central nervous system [10]. It has been established that IGF-1 
deficiency is causally related to the cognitive impairment of adults and the rodent models of aging [11].

In addition, clinical and experimental observations have emphasized that estrogen therapy exerts a positive effect on learning and memory functions [12]. Nonetheless, these benefits of estrogen for the brain are offset by the negative severe risks due to its oncogenic and proliferative effects on the breast and the endometrium [13]. Numerous studies have been performed to find an alternative for estrogen with less or no side effects. Regular exercise is a non-pharmacological intervention which is claimed to boost cognitive tasks in postmenopausal women [9].

Studies have demonstrated that exercise has modulatory effects on microRNAs, thereby improving BDNF and IGF-1 expression [1]. Furthermore, genistein, as a phytoestrogen, has similar estrogenic functions in neuronal protection with minor side effects. It has been demonstrated that genistein improves hippocampus neuronal proliferation and cell viability in vitro. These neuroprotective properties are mediated by the BDNF-Trk pathway [14]. Nevertheless, to the best of our knowledge, the effects of swimming or genistein and both of them on the expression of microRNA-132, IGF-1, and BDNF within the hippocampus of diabetic ovariectomized rats have not been investigated yet.

\section{Objectives}

In light of the aforementioned issues, the present study aimed to assess the effects of swimming and genistein on microRNA-132, IGF-1, and BDNF appearance, as well as spatial memory, within the hippocampus of ovariectomized diabetic rats.

\section{Materials and Methods}

Animals

Wistar female rats (10-week-old, weighting 180-220 g, $n=60$ ) were obtained from the Experimental Animal Research Center, Medical Faculty, Tabriz University, Tabriz, Iran. All rats were retained under well-ordered conditions (temperature $22-24^{\circ} \mathrm{C}$ with 12:12 dark-light cycle) and received a normal diet and ad lib water. The research was approved by the University Ethics Committee.

\section{Design of Experiment}

Animals were assigned to six groups $(n=10)$ as follows:

1. The sham control group underwent surgery without ovariectomy.

2. The OVX group were subjected to bilateral ovariectomy.
3. The OVX.D group was subjected to the induction of type 2 diabetes 10 days after bilateral ovariectomy.

4. The OVX.D.S group included animals that were treated with swimming, along with type 2 diabetes induction, 10 days after ovariectomy.

5. The OVX.D.G group received genistein (1 $\mathrm{mg} / \mathrm{Kg} /$ day; Sub Cutaneous) through the induction of type 2 diabetes 10 days after ovariectomy.

6. The OVX.D.G.S group was treated with both genistein and swimming.

Instruction of Ovariectomy

For this procedure, the animals were initially anesthetized (5 mg/kg xylazine and $50 \mathrm{mg} / \mathrm{kg}$ ketamine), and a small abdominal cut was made. The ovaries were then situated, and a silk cord was firmly tied to the oviduct and ovarian blood vessels. The oviduct was split, and the ovary was detached; thereafter, the muscle wall and skin were sutured with silk cord $[1,15]$.

\section{Instruction of type 2 diabetes}

To induce diabetes, 10 days after ovariectomy, diabetic groups were nourished by a high-fat diet (25\% protein, $17 \%$ carbohydrate, and $58 \%$ fat), and ad lib for the initial retro of eight weeks. Thereafter, a low dose of STZ (35 mg/ kg) was injected intraperitoneally in a citrate $0.1 \mathrm{M}$ buffer with $\mathrm{pH}=$ 4.5 [16]. The fasting glucose level in all rats after the generation of type 2 diabetes was measured by the Accu-Chek Active blood glucose meter. Animals with levels of glucose higher than $250 \mathrm{mg} \cdot \mathrm{dl}^{-1}$ were selected as diabetic rats [1].

\section{Genistein administration protocol}

Genistein was injected into the animals (one $\mathrm{mg} / \mathrm{kg} /$ day; Sub Cutaneous). [Sigma Chemical Incorporation (St. Louis, MO, USA)] [17].

\section{Swimming protocol}

After the recovery period of ovariectomy, animals were habituated with a swimming bath (5-20 $\mathrm{min} /$ day) for five consecutive days. Thereafter, trained animals swam for six successive days (60 min/day) for eight weeks, while Sham control group rats failed to swim. The exercised rats were studied $24 \mathrm{~h}$ after their latest exercise session [1].

\section{Morris Water Maze}

Spatial learning and memory were determined by the Morris Water Maze (MWM) task. The water maze was a dark round pool with a height of 100 $\mathrm{cm}$, a diameter of $136 \mathrm{~cm}$, and full of water $\left(20 \pm 1^{\circ} \mathrm{C}\right)$ to a depth of $60 \mathrm{~cm}$. The maze was conceptually allocated into four quadrants and release points that were marked as $\mathrm{N}, \mathrm{E}, \mathrm{S}$, and $\mathrm{W}$. 
A hidden square platform $(10-10 \mathrm{~cm})$ was placed within the center of the southwest quadrant which was submerged $1.5 \mathrm{~cm}$ below the surface of the water. Fixed additional visual signs were present at various locations all over the maze. A video camera was attached above the middle of the maze to record and observe the motions of rats. This technique was used to measure the escape latency, traveled path, and swimming speed [1].

Total RNA extraction and real-time polymerase chain reaction

The whole RNA, including messenger RNA and microRNA, was taken out from the hippocampus by RNX-Plus resolution kit (Fermentase, Cinagen Co. Iran) and miR-amp kit (Parsgenome Co. Iran) correspondingly in agreement to the manufacturer's orders. The expression of BDNF, IGF-1, and microRNA-132 genes was quantitatively measured by a real-time polymerase chain reaction (real-time PCR). The sequences of primers for every gene are presented in Table 1. The number of PCR products was standardized for the housekeeping gene glyceraldehyde-3phosphate dehydrogenase (GAPDH), mRNA samples, and microRNA-191 for microRNA samples (internal control).

\section{Statistical analysis}

Assessment of groups was accomplished by Tukey test two-way Analysis of variance (ANOVA). All analyses were performed in SPSS software (version 16). A p-value of less than 0.05 was considered statistically significant. The results were presented as means \pm SEM.

Table1. Sequences of primers for genes

\begin{tabular}{lcc}
\hline Genes & Accession number & Primers Sequence $^{\text {a }}$ \\
\hline IGF-1 & NM- 001082477 & F: AAG CCT ACA AAG TCA GCT CG \\
R: GGT CTT GTT TCC TGC ACT TC & F: GCGGCAGATAAA AAGACT GC \\
RDNF & NM_ 012513 & GCAGCCTTCCTTCGTGTA AC \\
GAPDH & NM_CCGCCTGGAGAAACCTGC \\
miR-132 & & R: TGAGAGCAATGCCAGCCCCA \\
miR-191a & TIMAT0017123 & Target sequence b \\
\hline
\end{tabular}

a Sequences were derived from NCBI (www.ncbi.nlm.nih.gov)

b Sequences were derived from miRBase (www.mirbase.org)

\section{Results}

MicroRNA-132 expression

As illustrated in Figure 1, the microRNA-132 appearance level was meaningfully diminished in the hippocampus of the OVX group, in comparison with that in the sham group $(\mathrm{P}<0.05)$, and this decrease was more pronounced in the OVX.D group, as compared to the sham group $(\mathrm{P}<0.01)$. Moreover,
Genistein and swimming treatment in OVX.D group significantly increased the microRNA-132 expression associated with OVX and OVX.D groups $(\mathrm{P}<0.05)$. The combined use of swimming and genistein augmented hippocampal expression of microRNA132, as compared to other groups (OVX, OVX.D, OVX.D.S, OVX.D.G), and this increase was statistically significant $(\mathrm{P}<0.05)$.

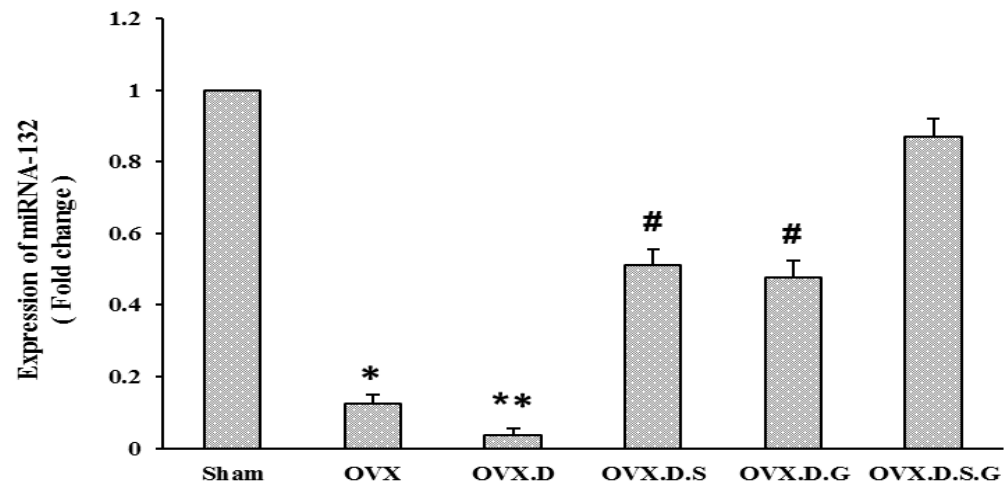

Figure 1. MicroRNA-132 expression in the hippocampus of the studied groups

OVX: ovariectomized group, OVX.D: ovariectomized with type 2 diabetic group, OVX.D.E: ovariectomized diabetic group with eight-week exercise. OVX.D.G: ovariectomized diabetic group with eight-week genistein injection. OVX.D.G.E: ovariectomized diabetic group with eight-week swimming training and genistein injection. Data are stated as mean \pm SEM.

* Significant differences, compared to the Sham, OVX.D, and treatment groups $(\mathrm{P}<0.05)$

** Significant differences, in comparison with Sham, OVX, and treatment groups $(P<0.05)$

\# Significant differences, compared to Sham, OVX, OVX.D, and OVX.D.G.S groups $(\mathrm{P}<0.05)$ 

Expression of brain-derived neurotrophic factor The expression of BDNF is displayed in Figure 2. The expression level of BDNF was meaningfully reduced in the hippocampus of the OVX group $(\mathrm{P}<0.05)$ and further declined in the OVX.D group, in comparison with the sham group $(\mathrm{P}<0.01)$. The expression of $\mathrm{BDNF}$ was meaningfully up-regulated in the hippocampus of OVX.D.S and OVX.D.G groups, as compared to that in OVX and OVX.D groups $(\mathrm{P}<0.05)$. The combined use of swimming and genistein improved hippocampal expression of BDNF, in comparison with OVX, OVX.D, OVX.D.S, OVX.D.G groups $(\mathrm{P}<0.05)$.

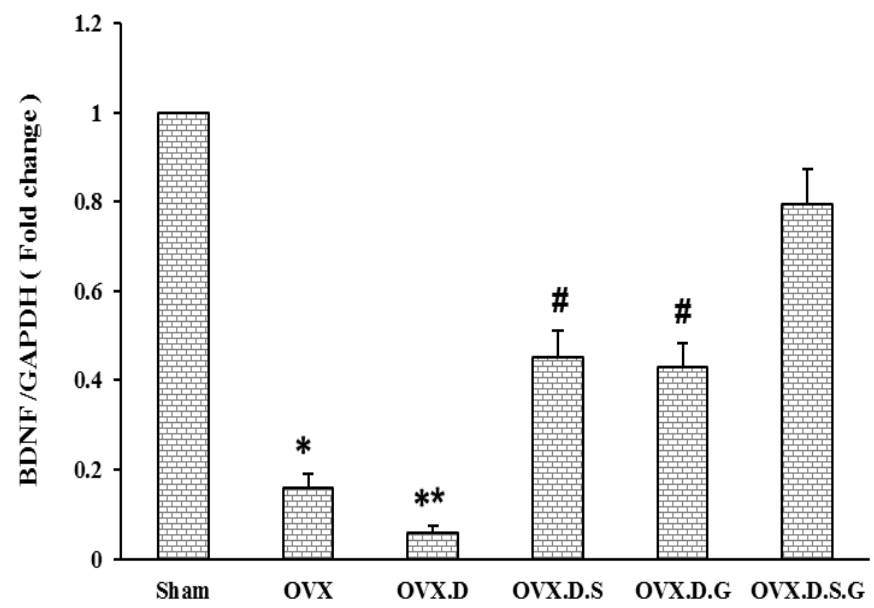

Figure 2. BDNF expression in the hippocampus of the studied groups

OVX: ovariectomized group, OVX.D: ovariectomized with type 2 diabetic group, OVX.D.E: ovariectomized diabetic group with eight-week exercise. OVX.D.G: ovariectomized diabetic group with eight-week genistein injection. OVX.D.G.E: ovariectomized diabetic group with eight-week swimming training and genistein injection. Data are stated as mean \pm SEM.

* Significant differences, compared to the Sham, OVX.D, and treatment groups $(\mathrm{P}<0.05)$

** Significant differences, in comparison with Sham, OVX, and treatment groups $(\mathrm{P}<0.05)$

\# Significant differences, compared to Sham, OVX, OVX.D, and OVX.D.G.S groups $(\mathrm{P}<0.05)$

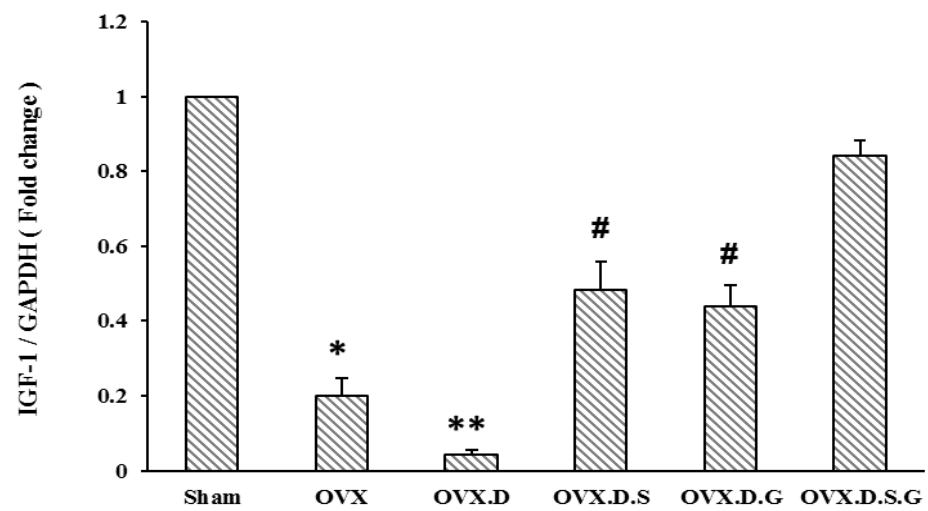

Figure 3. Expression of insulin growth factor 1 in the hippocampus of the studied groups

OVX: ovariectomized group, OVX.D: ovariectomized with type 2 diabetic group, OVX.D.E: ovariectomized diabetic group with eight-week exercise. OVX.D.G: ovariectomized diabetic group with eight-week genistein injection. OVX.D.G.E: ovariectomized diabetic group with eight-week swimming training and genistein injection. Data are stated as mean \pm SEM.

* Significant differences, compared to the Sham, OVX.D, and treatment groups $(\mathrm{P}<0.05)$

** Significant differences, in comparison with Sham, OVX, and treatment groups $(\mathrm{P}<0.05)$

\# Significant differences, compared to Sham, OVX, OVX.D, and OVX.D.G.S groups $(\mathrm{P}<0.05)$

\section{Expression of insulin growth factor 1}

The expression of IGF-1 is depicted in Figure 3. The IGF-1 expression level was meaningfully reduced in the hippocampus of the OVX group, as compared to that in the sham group $(\mathrm{P}<0.05)$, and this reduction was more pronounced in the OVX.D group, in comparison with the sham group $(\mathrm{P}<0.01)$. Moreover, the result demonstrated that exercise training and genistein up-regulated the expression of IGF-1 in the OVX.D group, in comparison with with OVX and OVX.D groups $(\mathrm{P}<0.05)$, and this upregulation was more pronounced in the hippocampus of the OVX.D.G.S group, as compared to other ovariectomized animals $(\mathrm{P}<0.05)$. 
Effect of swimming and genistein on spatial memory in different groups

Swimming and genistein treatment decreased path length (Figure 4A), travel latencies of searching for the hidden platform on acquisition trial (Figure 4B), and an increased percentage of spent time in the target quadrant (Figure.4C), and this variance was statistically significant $(\mathrm{P}<0.05)$. There were no significant changes in swimming speed among all exercised groups (Figure 4D).
Ovariectomy significantly impaired spatial learning performance in the MWM $(\mathrm{P}<0.05)$, and this impairment was more noticeable in the OVX.D group $(\mathrm{P}<0.05)$. Genistein and swimming enhanced spatial learning in OVX rats, in comparison with OVX and OVX.D groups $(\mathrm{P}<0.05)$. The improvement of spatial learning in the OVX.D.G.S group was within the range of the sham group. There was no important variance among the groups in swimming speed.
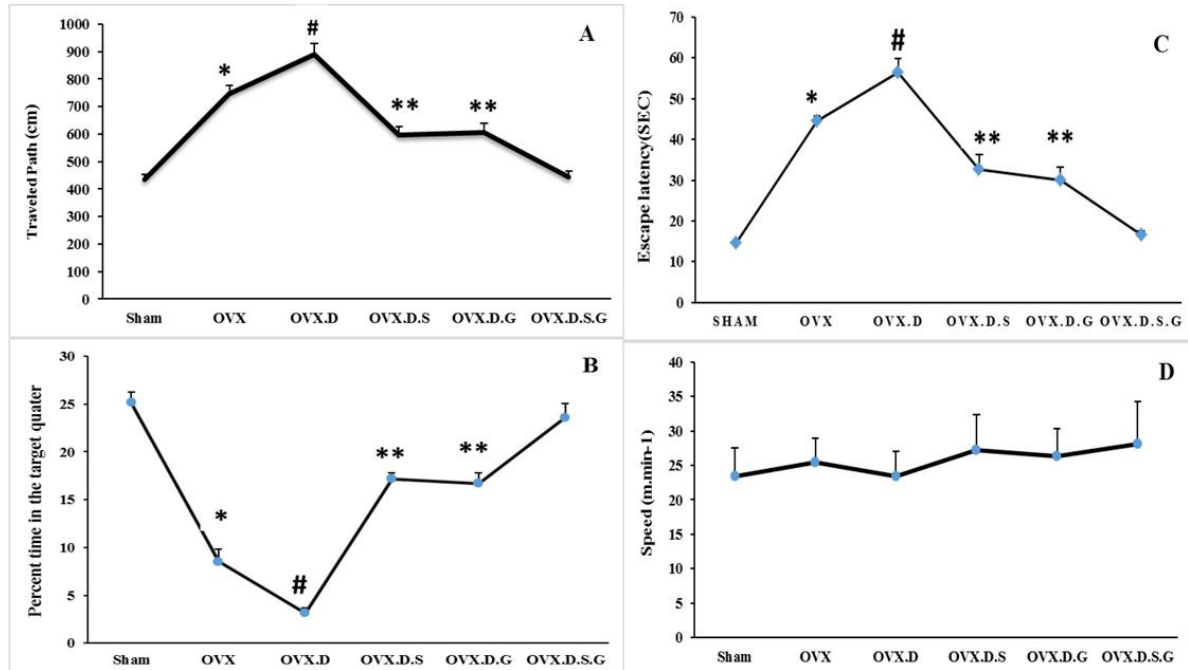

Figure 4. Effects of genistein and/or exercise on learning acquisition as measured through escape latencies (A), memory retention during the probe trial (B), the percentage of time spent in the target quadrant $(C)$, and swimming speed (D) detected in the MWM. Ovariectomy meaningfully decreased spatial learning presentation in the MWM $(\mathrm{P}<0.05)$, and this impairment was more pronounced in the OVX.D group $(\mathrm{P}<0.05)$. Spatial learning in OVX rats was also improved by the exercise and genistein injection in OVX.D, in comparison with OVX.D and OVX groups $(\mathrm{P}<0.05)$. In OVX.D.G.E group, spatial learning improved and no significant difference with Sham group. No significant difference was found among the groups in swimming speed.

OVX: ovariectomized group, OVX.D.E: diabetic ovariectomized group with eight-week exercise. OVX.D.G: diabetic ovariectomized group with eight-week genistein injection. OVX.D.G.E: diabetic ovariectomized group with eight-week swimming and genistein injection. Data are stated as mean \pm SEM.

* Significant differences, compared to the Sham, OVX.D, and treatment groups $(P<0.05)$

** Significant differences, in comparison with Sham, OVX, and treatment groups $(\mathrm{P}<0.05)$

\# Significant differences, compared to Sham, OVX, OVX.D, and OVX.D.G.S groups $(\mathrm{P}<0.05)$

\section{Discussion}

The present study was the first to determine whether exercise and/or genistein play a role in appearance changes of microRNA-132, IGF-1, and BDNF in the hippocampus of diabetic ovariectomized rats. Therefore, in this research, rat models of ovariectomy, diabetic ovariectomized rats, and diabetic ovariectomized rats that were treated with eight weeks of swimming and/or genistein administration were compared with the sham group. The major findings were as follows: 1) ovariectomized rats with or without diabetes markedly down-regulated microRNA-132, and 2) swimming and genistein increased the expression of these biomarkers; however, the combined use of them had a more significant effect.

The studies in the last few years have focused on the analysis of variance expression of microRNAs in heart failure, and the key part of the mammalian genome is controlled by microRNAs with great tissue specificity [4]. Synaptic smoothness, neuronal cell growth, swelling, and angiogenesis are associated with microRNA-132 [18]. A large amount of data, in vivo and in vitro, support the role of microRNA-132 in the mechanisms of memory formation and activity-stimulated gene expression [19]. Nevertheless, the expression of microRNA-132, as well as the effect of genistein and/or swimming in the hippocampus of the diabetic ovariectomized rat, has not been studied yet.

Ang (2006) pointed out that ovariectomy decreased the latency and distance to obtain platform in Morris Water Maze; nonetheless, this reduction was significantly more pronounced in the diabetic group. The studies have suggested that the irregular microRNAs expression is also involved in the pathogenesis processes in menopause and diabetic neuropathy, such as miRNA-1, -133, -141, -206, $223,-373$, and $-132[20,21]$. It seems that 
microRNAs control neuronal survival, synaptic plasticity, and neurotransmitter secretion through numerous mechanisms. It has been shown that BDNF and IGF-1 can increase microRNA-132 expression [22]. The BDNF improves memory and plays a key role in the expansion of dendritic spines, differentiation, and survival for extensive types of neuronal cells [23].

Furthermore, IGF-1 is associated with pro-insulin, is an effective factor for oligodendrocytes and neurons, as well as neural elaboration, differentiation, and growth [19,24]. A large amount of data, in vivo and in vitro, support the role of microRNA-132 in the mechanisms of memory formation and activity-stimulated gene expression [19]. Nevertheless, the expression of microRNA132, as well as the effect of swimming and/or genistein treatment in the hippocampus of the diabetic ovariectomized rat, has not been studied yet. Similar to other studies, the current research indicated that using Morris water maze protocol, ovariectomy increased the latency and distance to the obtain platform, compared to sham rats, and this increase was significantly more pronounced in the diabetic group. Moreover, to the best of our knowledge, for the first time, the obtained data demonstrated that the microRNA-132, IGF-1, and $\mathrm{BDNF}$ expression is down-regulated in the hippocampus of ovariectomized rats with or without diabetes.

Hormone therapy, such as estrogen, improves cognition and stops the progress of dementia in postmenopausal women, while other studies have not established the benefit of estrogen application $[25,26]$. In addition, the results of extensive investigational trials have indicated that hormone replacement treatments, such as estrogenalone therapy, increase the risk of breast cancer in postmenopausal women [9,27]. A great deal of effort has been made to prevent cognitive dysfunction, as well as learning and memory failures, in postmenopausal women mostly with diabetes, among which swimming and genistein have been recommended as substitutes for estrogen replacement to stop cognitive failure $[9,14]$.

In the present study, swimming and genistein treatment for eight weeks improved spatial memory, and this improvement was significantly more preannounced in the combined use of swimming and genistein treatment, as compared to OVX with or without diabetes group. In this study, eight weeks of regular swimming enhanced the expression of microRNA-132, IGF-1, and $\mathrm{BDNF}$ in the ovariectomized diabetic rats, in comparison with other groups. In addition, eight weeks of swimming enhanced spatial learning and memory on the MWM task in OVX.S, as compared to OVX animals.

The findings of the present study confirm the prior investigation, indicating that exercise training can disturb the risk factors of cognitive dysfunction. Cell proliferation can be enhanced by exercise which can inhibit apoptosis in the hippocampus and improves cognitive function despite estrogen deficiency [28]. Exercise can raise the expressions of IGF-1 and BDNF by increasing the expression of microRNA-132. Moreover, the expression of microRNA-132, IGF-1, and BDNF was boosted by eight weeks of genistein injection in the ovariectomized rats. In this study, genistein administration enhanced spatial learning and memory on the MWM task in OVX.G, as compared to OVX animals.

It has been proven that genistein, which has estrogenic and anti-diabetic effects, could be used as a substitute for estradiol to protect from central nervous system degeneration in diabetic postmenopausal women. The addition of genistein to the diet of ovariectomized rats enhanced the proteins in the brain [29]. Moreover, studies have pointed to the special effects of genistein on microRNAs [1]. Phytoestrogens, such as soy, improve cognition, which might be associated with the increase in the expression of BDNF and synaptogenic proteins in the hippocampus of ovariectomized rats [30].

The effects of daidzein and genistein on the expression of BDNF and hippocampus neuronal cell proliferation have been studied [31]. Furthermore, the combined use of exercise and genistein prevents cognitive impairment risk factors by increasing microRNA-132 gene expression, which may be related to an increase in IGF-1 and BDNF expressions in diabetic ovariectomized rats, as compared to other groups. The obtained data indicated that in estrogen-deficient states with diabetes, regular moderate exercise combined with genistein administration is the most effective treatment for cognitive function disturbances.

\section{Conclusions}

The results of the current study pointed to the beneficial effects of the combined use of swimming and genistein treatment on the improvement of spatial memory in OVX diabetic rats. These beneficial effects could also be caused by the increased microRNA-132 expression which is related to the up-regulation of IGF-1 and BDNF genes within the hippocampus of rats. Furthermore, a combination of exercise and genistein appears to be highly effective in inhibiting the expansion of cognition dysfunction. 


\section{Acknowledgments}

The funding for this study was supported by the Drug Applied Research Center of Tabriz University of Medical Sciences, Tabriz, Iran.

\section{Conflicts of Interest}

The authors declare that they have no conflict of interest.

\section{References}

1. Habibi P, Babri S, Ahmadiasl N, Yousefi H. Effects of genistein and swimming exercise on spatial memory and expression of microRNA 132, BDNF, and IGF-1 genes in the hippocampus of ovariectomized rats. Iranian Journal of Basic Medical Sciences. 2017; 20(8):856-62. [DOI:10.22038/IJBMS.2017.9106] [PMID] [PMCID]

2. Zhou Q, Lv D, Chen $\mathrm{P}, \mathrm{Xu} \mathrm{T}, \mathrm{Fu} \mathrm{S}$, Li J, et al. MicroRNAs in diabetic cardiomyopathy and clinical perspectives. Frontiers in Genetics. 2014; 5:185. [DOI:10.3389/fgene.2014.00185] [PMID] [PMCID]

3. Zhao Z, Wang H, Jessup JA, Lindsey SH, Chappell MC, Groban L. Role of estrogen in diastolic dysfunction. American Journal of Physiology. Heart and Circulatory Physiology. 2014; 306(5):H628-40. [DOI:10.1152/ajpheart.00859.2013] [PMID] [PMCID]

4. Felekkis K, Touvana E, Stefanou C, Deltas C. microRNAs: a newly described class of encoded molecules that play a role in health and disease. Hippokratia. 2010; 14(4):236-40. [PMID] [PMCID]

5. Liu Q, Du GQ, Zhu ZT, Zhang C, Sun XW, Liu JJ, et al. Identification of apoptosis-related microRNAs and their target genes in myocardial infarction post-transplantation with skeletal myoblasts. Journal of Translational Medicine. 2015; 13(1):270. [DOI:10.1186/s12967-015-0603-0] [PMID] [PMCID]

6. Shantikumar S, Caporali A, Emanueli C. Role of microRNAs in diabetes and its cardiovascular complications. Cardiovascular Research. 2012; 93(4):583-93. [DOI: 10.1093/cvr/cvr300] [PMID] [PMCID]

7. Li AY, Yang Q, Yang K. miR-133a mediates the hypoxiainduced apoptosis by inhibiting TAGLN2 expression in cardiac myocytes. Molecular and Cellular Biochemistry. 2015; 400(1-2):173-81. [DOI:10.1007/s11010-014-2273-2] [PMID]

8. Scharfman HE, Mercurio TC, Goodman JH, Wilson MA, MacLusky NJ. Hippocampal excitability increases during the estrous cycle in the rat: a potential role for brainderived neurotrophic factor. The Journal of Neuroscience. 2003; 23(37):11641-52. [DOI:10.1523/JNEUROSCI.2337-11641.2003] [PMID] [PMCID]

9. Saadati H, Sheibani V, Esmaeili-Mahani S, DarvishzadehMahani F, Mazhari S. Prior regular exercise reverses the decreased effects of sleep deprivation on brain-derived neurotrophic factor levels in the hippocampus of ovariectomized female rats. Regulatory Peptides. 2014; 194-195:11-5. [DOI:10.1016/j.regpep.2014.11.004] [PMID] [PMCID]

10. Grunbladh A, Johansson J, Nustl A, Nyberg F, Hallberg M. $\mathrm{GH}$ improves spatial memory and reverses certain anabolic androgenic steroid-induced effects in intact rats. The Journal of Endocrinology. 2013; 216(1):31-41. [DOI:10.1530/JOE12-0315] [PMID]

11. Deak F, Sonntag WE. Aging, synaptic dysfunction, and insulin-like growth factor (IGF)-1. The Journals of Gerontology. Series A, Biological Sciences and Medical Sciences. 2012; 67(6):611-25. [DOI:10.1093/gerona/gls118] [PMID] [PMCID]

12. Luine VN. Estradiol and cognitive function: past present and future. Hormones and Behavior. 2014; 66(4):602-18. [DOI:10.1016/j.yhbeh.2014.08.011] [PMID] [PMCID]

13. Menze ET, Esmat A, Tadros MG, Abdel-Naim AB, Khalifa AE. Genistein improves 3-NPA-induced memory impairment in ovariectomized rats: impact of its antioxidant, anti-inflammatory and acetylcholinesterase modulatory properties. PLoS One. 2015; 10(2):e0117223. [DOI: 10.1371/journal.pone.0117223] [PMID] [PMCID]

14. Peng $Y$, Jiang $B$, Wu $H$, Dai $R$, Tan L. Effects of genistein on neuronal apoptosis, and expression of $\mathrm{BCl}-2$ and Bax proteins in the hippocampus of ovariectomized rats. Neural Regeneration Research. 2012; 7(36):2874-81. [DOI: 10.3969/j.issn.1673-5374.2012.36.004] [PMID] [PMCID]

15. Habibi P, Alihemmati A, NourAzar A, Yousefi H, Mortazavi S, Ahmadiasl N. Expression of the Mir-133 and Bcl-2 could be affected by swimming training in the heart of ovariectomized rats. Iranian Journal of Basic Medical Sciences. 2016; 19(4):381-7. [PMID] [PMCID]

16. Daghigh F, Alihemmati A, Karimi P, Habibi P, AhmadiasI N. Genistein preserves the lungs of ovariectomized diabetic rats: addition to apoptotic and inflammatory markers in the lung. Iranian Journal of Basic Medical Sciences. 2017; 20(12):1312-7. [DOI:10.22038/IJBMS.2017.9599] [PMID] [PMCID]

17. Yousefi $H$, Karimi $P$, Alihemmati A, Alipour MR, Habibi $P$, Ahmadiasl $N$. Therapeutic potential of genistein in ovariectomy-induced pancreatic injury in diabetic rats: the regulation of MAPK pathway and apoptosis. Iranian Journal of Basic Medical Sciences. 2017; 20(9):1009-15. [DOI:10.22038/IJBMS.2017.9269] [PMID] [PMCID]

18. Hancock ML, Preitner N, Quan J, Flanagan JG. MicroRNA-132 is enriched in developing axons, locally regulates Rasa1 mRNA, and promotes axon extension. The Journal of Neuroscience. 2014; 34(1):66-78. [DOI:10.1523/JNEUROSCI.3371-13.2014] [PMID] [PMCID]

19. Wang RY, Phang RZ, Hsu PH, Wang WH, Huang HT, Liu IY. In vivo knockdown of hippocampal miR- 132 expression impairs memory acquisition of trace fear conditioning. Hippocampus. 2013; 23(7):625-33. [DOI:10.1002/hipo.22123] [PMID]

20. Fernandez-Valverde SL, Taft RJ, Mattick JS. MicroRNAs in $\beta$ cell biology, insulin resistance, diabetes and its complications. Diabetes. 2011; 60(7):1825-31. [DOI: 10.2337/db11-0171] [PMID] [PMCID]

21. Stachowiak G, Zając A, Nowak M, Stetkiewicz T, Wilczyński JR. Hemostatic disorders of the menopausal period: the role of microRNA. Przeglad Menopauzalny. 2015; 14(2):144-8. [DOI:10.5114/pm.2015.52155] [PMID] [PMCID]

22. Li Y, Kannan G, Pletnikov MV, Yolken RH, Xiao J. Chronic infection of Toxoplasma gondii downregulates miR-132 expression in multiple brain regions in a sexdependent manner. Parasitology. 2015; 142(04):623-32. [DOI:10.1017/S003118201400167X] [PMID] [PMCID]

23. Murray PS, Holmes PV. An overview of brain-derived neurotrophic factor and implications for excitotoxic vulnerability in the hippocampus. International Journal of Peptides. 2011; 2011:654085. [DOI:10.1155/2011/654085] [PMID] [PMCID]

24. Morović S, Demarin V. Role of physical activity on human brain functions. Periodicum Biologorum. 2014; 116(2): 219-21.

25. Yaffe K, Sawaya G, Lieberburg I, Grady D. Estrogen therapy in postmenopausal women: effects on cognitive function and dementia. JAMA. 1998; 279(9):688-95. [DOI:10.1001/jama.279.9.688] [PMID]

26. Janicki SC, Schupf N. Hormonal influences on cognition and risk for Alzheimer's disease. Current Neurology and Neuroscience Reports. 2010; 10(5):359-66. [DOI:10.1007/s11910-010-0122-6] [PMID] [PMCID]

27. Lovekamp-Swan T, Glendenning ML, Schreihofer DA. A high soy diet enhances neurotropin receptor and $\mathrm{BCl}-\mathrm{X} \mathrm{L}$ gene expression in the brains of ovariectomized female rats. Brain Research. 2007; 1159:54-66. [DOI:10.1016/j.brainres.2007.05.026] [PMID] [PMCID]

28. Kim TW, Kim CS, Kim JY, Kim CJ, Seo JH. Combined exercise ameliorates ovariectomy-induced cognitive impairment by enhancing cell proliferation and suppressing apoptosis. Menopause. 2016; 23(1):18-26. [DOI 10.1097/GME.0000000000000486] [PMID]

29. Lee J, Cho HS, Kim DY, Cho JY, Chung JS, Lee HK, et al. Combined effects of exercise and soy isoflavone diet on paraoxonase, nitric oxide and aortic apoptosis in ovariectomized rats. Appetite. 2012; 58(2):462-9. [DOI:10.1016/j.appet.2011.12.015] [PMID]

30. Pan M, Li Z, Yeung V, Xu RJ. Dietary supplementation of soy germ phytoestrogens or estradiol improves spatial 
memory performance and increases gene expression of BDNF, TrkB receptor and synaptic factors in ovariectomized rats. Nutrition \& Metabolism. 2010; 7(1):75. [DOI: 10.1186/1743-7075-7-75] [PMID] [PMCID]

31. Pan M, Han H, Zhong C, Geng Q. Effects of genistein and daidzein on hippocampus neuronal cell proliferation and BDNF expression in H19-7 neural cell line. The Journal of Nutrition, Health \& Aging. 2012; 16(4):389-94. [DOI:10.1007/s12603-011-0140-3] [PMID] 